\title{
Pacientes con ERC estadio 5: dieta baja en proteínas, cetoanálogos y aminoácidos esenciales
}

\author{
Gabriela Pomiglio ${ }^{1}$, María A. Aimar ${ }^{2 *}$, María L. Ocampo ${ }^{3}$, Agustina Heredia ${ }^{3}$ - \\ Colaboradores del Grupo RIANA\#
}

${ }^{1}$ Servicio de Nutrición Renal, Aterym Córdoba, Aterym Alta Gracia, Provincia de Córdoba; ${ }^{2}$ Nutrihome, Buenos Aires; ${ }^{3}$ Servicio de Nefrología, Hospital Italiano de Buenos Aires, Buenos Aires. Argentina

\section{Resumen}

Objetivo: Analizar la evolución de pacientes con enfermedad renal crónica en estadio 5 durante un año, con dieta hipoproteica, cetoanálogos y aminoácidos esenciales. Métodos: Cohorte retrospectiva, de pacientes con enfermedad renal crónica en estadio 5, asistidos ambulatoriamente, con dieta hipoproteica $(0.6 \mathrm{~g} / \mathrm{kg} / \mathrm{dí}$ ) y cetoanálogos, durante un año. Variables: función renal, anemia, albuminemia, equilibrio electrolítico-mineral y estado nutricional cada seis meses. Resultados: Fueron incorporados 75 pacientes: 23 ingresaron a diálisis, cuatro se trasplantaron, siete suspendieron y uno falleció. Cuarenta pacientes fueron evaluados al año; el 35\% fueron mujeres. Media de ingesta proteica: 0.58 (desviación estándar [DE]: 0.06), 0.59 (DE: 0.05) y $0.58 \mathrm{~g} / \mathrm{kg} / \mathrm{día}$ (DE: 0.05) al inicio, 6 y 12 meses respectivamente. Ingesta cal/kg/d: 32.3 (DE: 3.37 ), 32.7 (DE: 4.5) y 31.9 (DE: 3.41) al inicio, 6 y 12 meses respectivamente. Todos recibieron cetoanálogos y aminoácidos esenciales. Los pacientes presentaron una caída leve pero significativa del filtrado glomerular (sexto al duodécimo mes): $13 \mathrm{ml} / \mathrm{min} / 1.73$ $\mathrm{m}^{2}$ (11.3-14.3) y $12.1 \mathrm{ml} / \mathrm{min} / 1.73 \mathrm{~m}^{2}$ (8.8-13.7) respectivamente $(p=0.0031)$. Uremia, calcemia, fosfatemia, potasemia, albuminemia, peso e índice de masa corporal no presentaron cambios significativos. Conclusión: La dieta hipoproteica suplementada con cetoanálogos en pacientes con enfermedad renal crónica estadio 5 permitió sostener el tratamiento conservador manteniendo el estado nutricional y equilibrio mineral.

Palabras clave: Enfermedad renal crónica. Dieta hipoproteica. Cetoanálogos. Tratamiento conservador.

\section{Stage 5 CKD: Low protein diet, ketoanalogues and essential aminoacids}

\section{Abstract}

Objective: To analize the evolution of patients with stage 5 chronic kidney disease (CKD 5) during a year, with hypoprotein diet, ketoanalogs and essential aminoacids. Methods: Retrospective cohort of patients with CKD 5, with a hypoprotein diet $(0.6 \mathrm{~g} / \mathrm{kg} / \mathrm{day})$ and ketoanalogues for 1 year. Variables: renal function, anemia, albuminemia, electrolyte-mineral balance and nutritional status. Results: 75 patients were recruited-23 admitted to dialysis, 4 transplanted, 7 suspended and one died. Forty patients were evaluated at one year. Protein intake: $0.58 \mathrm{~g} / \mathrm{kg} / \mathrm{day}$ (SD: 0.06$), 0.59$ (SD: 0.05) and 0.58 (SD: 0.05) at baseline, 6 and 12 months respectively-Cal/kg/d intake: 32.3 (SD: 3.37 ), 32.7 (SD: 4.5)

\section{Correspondencia:}

*Aimar M. Andrea

E-mail: maimar@nutrihome.com.ar
Fecha de recepción: 07-06-2021

Fecha de aceptación: 07-09-2021

DOI: 10.24875/NEFRO.21000021
Disponible en internet: 27-12-2021

Nefro Latinoam. 2021;18:111-118 www.nefrologialatinoamericana.com

2444-9032/@ 2021 Sociedad Latinoamericana de Nefrología e Hipertensión. Publicado por Permanyer. Este es un artículo open access bajo la licencia CC BY-NC-ND (http://creativecommons.org/licenses/by-nc-nd/4.0/). 
Nefro Latinoam. 2021;18

and 31.9 (SD: 3.41) at the beginning, 6 and 12 months respectively. All received ketoanalogos and essential aminoacids. The patients had a slight but significant drop in glomerular filtration rate (sixth to twelfth month): 13 ( $\mathrm{m} / \mathrm{min} / 1.73 \mathrm{~m}^{2}$ ) (11.3-14.3) and $12.1\left(\mathrm{ml} / \mathrm{min} / 1.73 \mathrm{~m}^{2}\right)(8.8-13.7)$ respectively $(p=0.0031)$. Uremia, calcium, phosphataemia, potassium, albuminemia, weight and BMI did not show significant changes. Conclusion: Hypoprotein diet supplemented with ketoanalogs and essential aminacids in CKD 5 allowed to sustain conservative treatment while maintaining nutritional status and mineral balance.

Key words: Chronic kidney disease. Low protein diet. Ketoanalogues. Conservative treatment.

\section{Introducción}

La enfermedad renal crónica (ERC) afecta a más del $10 \%$ de la población adulta en todo el mundo 1 . Se estima que un porcentaje de estos pacientes con ERC progresan a enfermedad renal crónica terminal (ERCT), requiriendo de algún tipo de tratamiento sustitutivo como diálisis o trasplante de riñón. La morbilidad y mortalidad cardiovascular de la ERCT son excepcionalmente altas, con una supervivencia global a cinco años inferior al $50 \%$. El $10 \%$ de estos pacientes mueren en los primeros 90 días después de la transición de diálisis y más del $20 \%$ en el primer año'.

En Argentina se estima que la prevalencia de ERC es del $12.7 \%$, una de cada ocho personas adultas presentaría ERC. Este dato surge de la Segunda Encuesta Nacional de Nutrición y Salud (ENNyS 2) $2019^{2}$. La situación epidemiológica en el año 2019 muestra que en tratamiento sustitutivo renal se encuentran 41,223 pacientes: 10,923 trasplantados y 30,300 en diálisis crónica. En el año 2019, 7,310 pacientes ingresaron a tratamiento de reemplazo renal, 106 en trasplante anticipado y 7,204 en diálisis crónica ${ }^{3}$. La nefropatía diabética y la nefroangioesclerosis son la primera y segunda causa respectivamente de ingreso a diálisis. El porcentaje de los pacientes que presentan hipertensión arterial en su ingreso aumentó del $80.7 \%$ en 2004 al $83.4 \%$ en $2019^{3}$.

Respecto del estado nutricional, el $48.2 \%$ de los pacientes llega a su primera diálisis con valores de albuminemia $<3.5 \mathrm{~g} / \mathrm{dl}$, siendo este un predictor de mayor mortalidad. La prevalencia de población con sobrepeso-obesidad llega al $57 \%{ }^{3}$. Este incremento del peso en desmedro del peso normal está en directa relación con un estado de malnutrición ${ }^{3}$. La intervención nutricional es una piedra angular, siendo la implementación de dietas restringidas en proteínas un desafío en la práctica clínica y una estrategia nutricional bien conocida para mejorar el perfil metabólico de pacientes con ERC moderada a avanzada 4 .
Una forma de proteger la función renal y ralentizar la progresión de la enfermedad renal es reducir la hiperfiltración e hipertrofia glomerular reduciendo la ingesta proteica ${ }^{5}$.

La dieta hipoproteica se ha utilizado desde hace años con el objetivo de prevenir la ERC, disminuir complicaciones como acidosis metabólica, alteración del metabolismo óseo-mineral, insulinorresistencia, proteinuria y deterioro de la función renal ${ }^{4-6}$. Existe consenso respecto al beneficio de la restricción proteica para retrasar la progresión de la enfermedad renal siempre que se provea un aporte adecuado de energía ${ }^{7,8}$.

La dieta baja en proteínas (DBP) considera un aporte de $0.55-0.6 \mathrm{~g} / \mathrm{kg} / \mathrm{día}$ y un adecuado aporte calórico (25-35 kcal/kg/día) para lograr un balance neutro de nitrógeno en pacientes metabólicamente estables ${ }^{4}$. La dieta muy baja en proteínas (DMBP) proporciona 0.3$0.4 \mathrm{~g} / \mathrm{kg} / \mathrm{día}$, necesariamente se debe suplementar ${ }^{9}$.

La suplementación de estas dietas con una mezcla de aminoácidos esenciales y cetoanálogos tiene como ventaja la reducción más marcada de los compuestos urémicos y, consecuentemente, de los síntomas urémicos. Además de mejorar la sensibilidad a la insulina, permite controlar la presión arterial, reducir la proteinuria, corregir la acidosis metabólica y los trastornos óseos y minerales, enlentecer la progresión del daño renal, retrasar el inicio de la diálisis y preservar el estado nutricional ${ }^{10-12}$.

Los suplementos juegan un importante rol para obtener efectos nutricionales beneficiosos y seguros. En este sentido, los aminoácidos esenciales y los cetoácidos son los suplementos utilizados en pacientes con ERC y regímenes de baja ingesta proteica para prevenir la malnutrición ${ }^{13}$. La razón del beneficio de los cetoanálogos y aminoácidos esenciales es que, para la mayoría de los aminoácidos esenciales, el primer paso en la degradación es la transaminación, que implica la eliminación del grupo amino (NH2) unido a un carbono y su reemplazo por un grupo ceto o hidroxi ${ }^{11}$. El cetoácido formado por transaminación puede degradarse 
aún más por oxidación. Por el contrario, los análogos de cetoácidos o hidroxiácidos de los aminoácidos esenciales pueden ser transaminados para formar nuevamente los respectivos aminoácidos esenciales. De modo que cuando una persona consume cetoácidos o hidroxianálogos de aminoácidos esenciales, algunos serán transaminados, aumentando los respectivos aminoácidos esenciales. Este proceso utiliza grupos amino circulantes, evitando así su incorporación a la urea u otros agentes potencialmente tóxicos, productos de desecho nitrogenados. La condición para que esto ocurra es la presencia de cantidades significativas de cetoanálogos o hidroxiácidos circulantes ${ }^{11}$.

El logro de los objetivos de dicho tratamiento demanda un seguimiento individual y periódico por parte del equipo de salud, con nefrólogos y nutricionistas capacitados en el área renal 7 . Se requiere la realización de entrevistas nutricionales periódicas que evalúen el estado nutricional del paciente apoyándolo y motivándolo con indicaciones que permitan alcanzar en forma gradual y en un determinado periodo el nivel óptimo de ingesta proteica ${ }^{3}$. En América Latina no encontramos comunicaciones científicas de experiencias sobre el uso de cetoanálogos en la ERC en estadio 5 con tratamiento conservador. El objetivo del presente estudio fue analizar por medio de una base de datos la evolución clínica de un grupo de pacientes con ERC en estadio 5 seguidos durante un año y tratados con una DBP y suplementada con aminoácidos esenciales y cetoanálogos de aminoácidos esenciales.

\section{Material y métodos}

\section{Diseño del estudio}

Estudio de cohorte retrospectivo, de pacientes seguidos durante un año de tratamiento con ERC estadio 5 sin diálisis (S/D). Se recolectaron los datos en servicios de nefrología de siete ciudades de Argentina (Córdoba, Ciudad Autónoma de Buenos Aires, Bahía Blanca, Neuquén, Mar del Plata, Santa Fe y Santa Rosa), por profesionales que integran el grupo RIANA (Red Interdisciplinaria de Atención Nutricional Ambulatoria) del mes de abril de 2016 a abril de 2019. Se seleccionaron estos centros por su modalidad de atención: equipo interdisciplinario (medico nefrólogo y nutricionista). Se incluyeron los datos de pacientes adultos mayores de 21 años de edad con ERC estadio 5 S/D. Para proteger la identidad de los registros de los participantes los datos personales fueron disociados e incorporados a una base de datos con un código.
Se consideraron criterios de exclusión los datos de pacientes con ERC en estadio 5 en tratamiento sustitutivo (hemodiálisis o diálisis peritoneal), oncológicos, inmunodeprimidos, embarazadas, mujeres en periodo de lactancia y aquellos que constase en los registros que no cumplieron con las indicaciones médicas $y / 0$ nutricionales.

La modalidad de atención de los centros participantes constatada en la revisión de la documentación consistió en un plan nutricional con un aporte de calorías según los requerimientos, con aporte proteico de $0.6 \mathrm{~g} / \mathrm{kg} / \mathrm{día}$ más la incorporación de un comprimido de alfacetoanálogos de aminoácidos esenciales (Ketosteril ${ }^{\circledR}$ Fresenius-Kabi, Alemania) cada 5 kg de peso ideal.

Se recuperó la información de todos los datos del seguimiento clínico, nutricional y control antropométrico.

Teniendo en cuenta los registros, las siguientes variables se estudiaron y analizaron a los 0,6 y 12 meses:

- Función renal. Se registraron los siguientes indicadores: filtrado glomerular $(\mathrm{FG})$, creatininemia (valores de referencia de creatinina: $0.7-1.3$ y $0.6-1.1 \mathrm{mg} / \mathrm{dl}$ para hombres y para mujeres, respectivamente) y uremia (valores de referencia: 20-45 mg/dl). La estimación de la tasa de FG (eTFG) se realizó mediante la ecuación MDRD (Modification of Diet in Renal Disease) $4^{14}$.

- Estado nutricional. Se utilizaron los siguientes indicadores: índice de masa corporal (IMC) (peso [kg]/ talla $^{2}$ [m]; valor normal: $\left.18-25 \mathrm{~kg} / \mathrm{m}^{2}\right)^{15}$, peso $(\mathrm{kg})$, albúmina (valor de referencia: $3.4-5.4 \mathrm{~g} / \mathrm{dl}$ ) y anemia (indicador: hemoglobina $[\mathrm{Hb}]<11 \mathrm{~g} / \mathrm{dl}$ ).

- Equilibrio electrolítico-mineral: calcio, fósforo y potasio. Para evaluar su evolución, se utilizaron los siguientes indicadores: calcemia (valor de referencia: 8.5-10.5 mg/dl), Fostafemia (valor de referencia: 2.5$5.6 \mathrm{mg} / \mathrm{dl})$ y potasemia $(3.5-5.1 \mathrm{mEq} / \mathrm{l})$.

Las determinaciones de laboratorio registradas en las historias clínicas se realizaron en los centros de nefrología participantes con los siguientes métodos: Jaffé automatizado (creatinina), cinético UV automatizado (uremia y fósforo sérico), electroforesis ( $\mathrm{Hb}$ y albúmina), método colorimétrico automatizado (calcio sérico) y electrodo ion selectivo (potasio).

\section{Análisis estadístico}

Se evaluó la normalidad de los datos y se expresaron como media y desviación estándar (DE) o mediana y rango intercuartílico, según correspondiera. 
Se compararon las medias al año y al inicio del estudio mediante la prueba $t$ de Student pareada o la prueba de Wilcoxon de rangos signados, según correspondiera.

En cada uno de los tres puntos de evaluación clínica, se compararon las medias de FG, IMC, peso y de las concentraciones séricas de urea, creatinina, $\mathrm{Hb}$, potasio, calcio, fósforo y albúmina. Para las variables cuya distribución fue compatible con la normal se usó la prueba de ANOVA de medidas repetidas, mientras que para aquellas con distribución distinta de la normal se usó la prueba de Friedman. Cuando se encontró significancia estadística, la comparación de a pares post hoc se realizó mediante la prueba t de Student pareada - la prueba de Wilcoxon de rangos signados, según correspondiera, en ambos casos usando la corrección de Bonferroni.

El análisis de los datos obtenidos se realizó con el software SPSS versión 18.

\section{Consideraciones éticas}

Dado que se trata de un estudio observacional retrospectivo en el que se trabajó con una base de datos, no se requirió el consentimiento informado del paciente y el análisis estuvo exento de la aprobación de la junta de revisión institucional o del comité de ética.

Para proteger la identidad del registro de los participantes los datos personales fueron disociados e incorporados a otra base de datos con un código.

\section{Resultados}

Fueron incorporados los datos de 75 pacientes que cumplieron con los criterios de inclusión analizando su evolución durante 12 meses.

En la figura 1 se observa el diagrama de flujo y los motivos por los cuales se retiraron los pacientes del análisis.

A los 12 meses fueron evaluados 40 pacientes, de los cuales 14 (35\%) fueron de sexo femenino. La edad mediana fue de 65 años (55.5-72.3) con un mínimo de 24 años y un máximo de 87 años. El diagnostico etiológico correspondió en nueve pacientes a nefropatía diabética, nefroangioesclerosis en 13 , riñón senil uno, poliquistosis en cinco, dos de origen desconocido y otros diagnósticos en 10 pacientes.

Los pacientes adhirieron al tratamiento con DBP; la media de ingesta en $\mathrm{g} / \mathrm{kg} /$ día fue de 0.58 (DE: 0.06), 0.59 (DE: 0.05) y 0.58 (DE: 0.05) al inicio, 6 y 12 meses respectivamente. La ingesta calórica por $\mathrm{kg} / \mathrm{d}$ fue de

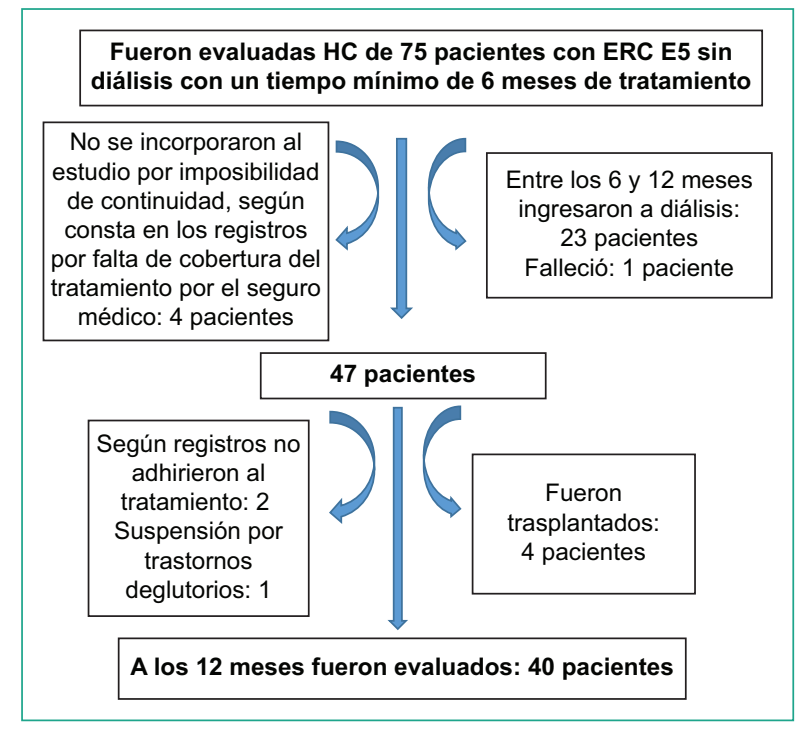

Figura 1. Diagrama de flujo de la evolución de los pacientes seleccionados.

HC: historia clínica; ERC: enfermedad renal crónica.

32.3 (DE: 3.37), 32.7 (DE: 4.5) y 31.9 (DE: 3.41) al inicio, 6 y 12 meses respectivamente. El número de comprimidos de Ketosteril ${ }^{\circledR}$ diarios recibidos por los pacientes a lo largo del seguimiento tuvo una mediana de 12

En la tabla 1 se observa el resumen general de la evolución de los pacientes.

En la figura 2 se observa la evolución de los filtrados glomerulares en los tres cortes presentados. En la FG se observó una disminución leve, aunque significativa entre el sexto y duodécimo mes. También se encontró un aumento significativo de la creatininemia.

No se hallaron diferencias significativas a lo largo de los tres cortes en el año de la uremia, la calcemia, fosfatemia y potasemia. También se mantuvieron sin cambios la albuminemia, el peso y el IMC (Tabla 1) (Fig. 3).

Cuando analizamos los pacientes según edad, tomando como referencia la mediana, en mayores o menores de 65 años, se observaron diferencias en el comportamiento. La filtración glomerular cae significativamente en los menores de 65 años: 13 (11.95-14), $12.25(11.23-14)$ y $10.95 \mathrm{ml} / \mathrm{min}(8-12.94)(p=0.014)$, al inicio, 6 y 12 meses respectivamente. En el grupo de los mayores de 65 años no hallamos cambios significativos desde el inicio hasta los 12 meses: 13.14 (11$14.10), 13.89(11.56-14.77)$ y $12.86 \mathrm{ml} / \mathrm{min}(10.58-14)$ $(p=0.853)$. 
Tabla 1. Evolución de las variables de función renal, anemia y estado nutricional al inicio, 6 y 12 meses

\begin{tabular}{|c|c|c|c|c|}
\hline & $\begin{array}{c}\text { Inicio } \\
\text { Mediana (IC 95\%) }\end{array}$ & $\begin{array}{c}6 \text { meses } \\
\text { Mediana (IC 95\%) }\end{array}$ & $\begin{array}{c}12 \text { meses } \\
\text { Mediana (IC 95\%) }\end{array}$ & Valor de $p$ \\
\hline Filtrado glomerular $\left(\mathrm{ml} / \mathrm{min} / 1.73 \mathrm{~m}^{2}\right)$ & $13(11-14)$ & $13(11.3-14.3)$ & $12.1(8.8-13.7)$ & 0.0031 \\
\hline Creatininemia (mg/dl) & $4.60(4.00-5.23)$ & $4.34(4.00-5.08)$ & $4.80(4.18-5.83)$ & 0.0001 \\
\hline Urea (mg/dl) & $119(102-165)$ & $126(99-152)$ & $136(117-168)$ & 0.1030 \\
\hline Hemoglobina (g/dl) & $11.8(10.1-12.4)$ & $11.6(10.5-12)$ & $11.2(10.4-12)$ & 0.0238 \\
\hline Calcemia (mg/dl) & $9.0(8.6-9.4)$ & $9.2(8.9-9.5)$ & $9.0(8.5-9.3)$ & 0.1080 \\
\hline Fósforo (mg/dl) & $4.3(4.0-4.6)$ & $4.2(3.8-4.9)$ & $4.4(4.0-5.3)$ & 0.9450 \\
\hline Potasio $\mathrm{mEq} / \mathrm{l}$ & $4.75 \pm 0.63$ & $4.66 \pm 0.65$ & $4.62 \pm 0.65$ & 0.3990 \\
\hline Albuminemia (g/dl) & $4.00(3.72-4.30)$ & $4.10(3.80-4.30)$ & $4.03(3.92-4.28)$ & 0.564 \\
\hline Peso (kg) & $77.1 \pm 21.4$ & $77.3 \pm 21.8$ & $77.9 \pm 22.1$ & 0.3350 \\
\hline IMC & $27.4 \pm 5.56$ & $27.4 \pm 5.57$ & $27.6 \pm 5.66$ & 0.3380 \\
\hline
\end{tabular}

IC: intervalo de confianza-IMC: índice de masa corporal.

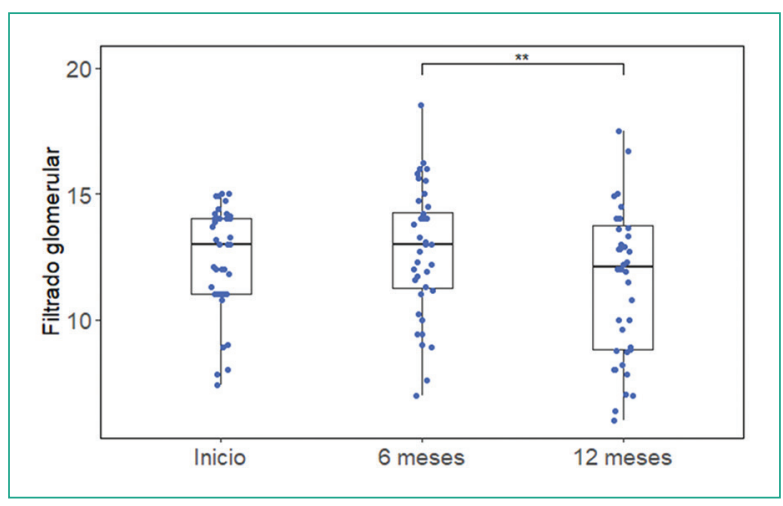

Figura 2. Filtración glomerular (ml/min) a los 3,6 y 12 meses de seguimiento.

${ }^{* *} p=0.00$.

También fue significativo el aumento de la creatininemia en menores de 65 años: 4.65 (4.21-5.35), $4.45(4.07-5.26)$ y $5.64 \mathrm{mg} / \mathrm{dl}(4.47-6.80)(p=0.002)$ al inicio, 6 y 12 meses respectivamente. En el caso de los mayores de 65 años el aumento fue menos marcado pero significativo: 4.20 (3.70-4.95), $4.20(4.00-4.84)$ y $4.70 \mathrm{mg} / \mathrm{dl}(3.77-4.93)(p=0.044)$.

La uremia aumentó significativamente en los menores de 65 años: 102 (94.5-163), 126 (94.5-151) y $126 \mathrm{mg} / \mathrm{dl}(113.5-174.5)(p=0.003)$ al inicio, a los 6 y 12 meses respectivamente. En los mayores de 65 años los cambios no fueron significativos.

También se observó que la fosfatemia aumentó de manera significativa, pasando de $4.20 \mathrm{mg} / \mathrm{dl}(4.07-4.79)$ al inicio a $4.25 \mathrm{mg} / \mathrm{dl}(4-4.77)$ a los 6 meses y $4.95 \mathrm{mg} / \mathrm{dl}$

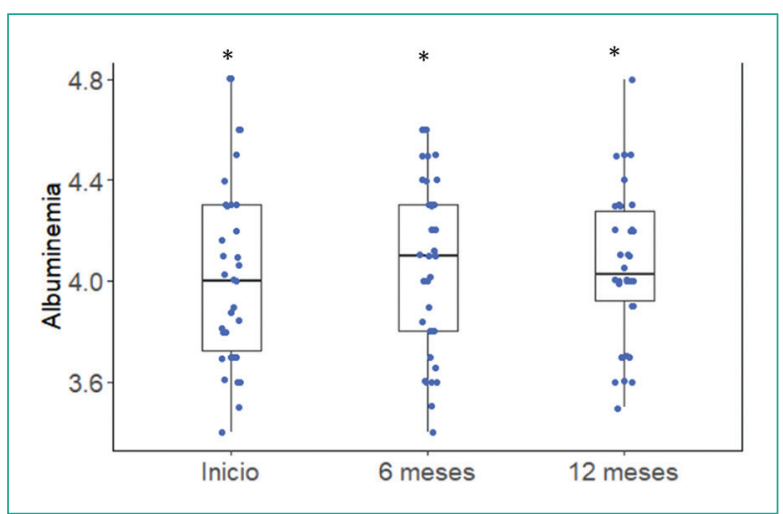

Figura 3. Albuminemia $(\mathrm{g} / \mathrm{dl})$ a los 3,6 y 12 meses de seguimiento. Valores graficados en medianas y rango intercuartílico.

*Valores graficados en Medianas y rango intercuartílico

(4.38-5.50) a los 12 meses ( $p=0.004)$. En cambio, en los mayores de 65 años mostró una disminución discreta pero significativa: 4.30 (4-4.55), 4.20 (3.80-4.90) y $4 \mathrm{mg} / \mathrm{dl}(3.85-4.60)(p=0.038)$.

En los demás indicadores ( $\mathrm{Hb}$, calcemia, potasemia, albuminemia, peso e IMC) no se observaron cambios significativos en ninguno de los dos grupos.

\section{Discusión}

En este estudio de cohorte retrospectivo encontramos que según los registros un alto porcentaje de pacientes en seguimiento con ERC en estadio 5 
por un equipo interdisciplinario de nefrólogos y nutricionistas, la implementación de un tratamiento con dieta controlada en proteínas y suplementada con cetoanálogos, permitió sostener el tratamiento conservador.

Estos pacientes no presentaron cambios significativos en su estado nutricional estimado por IMC y albuminemia. Si bien se observaron cambios significativos en la función renal, no afectaron la estabilidad clínica y metabólica de los pacientes, estimada por calcemia, fosfatemia, potasemia, albuminemia y manifestaciones clínicas.

Una reciente publicación muestra los resultados de un metaanálisis que incluye 951 pacientes con ERC, con eTFG mayor y menor de $18 \mathrm{ml} / \mathrm{min} / 1.73 \mathrm{~m}^{2}$ de 10 ensayos clínicos y dos estudios no controlados con dieta controlada en proteínas y cetoanálogos ${ }^{16}$. El uso de cetoanálogos revirtió significativamente la disminución de la tasa de FG. Los autores concluyen que la DBP con cetoanálogos no presenta diferencias significativas en los resultados respecto de la DMBP. En el grupo de los pacientes con $F G>18 \mathrm{ml} / \mathrm{min} / 1.73 \mathrm{~m}^{2}$ los resultados mostraron una mejoría significativa del filtrado con DBP suplementada con cetoanálogos. Pero en los pacientes con $\mathrm{FG}<18 \mathrm{ml} / \mathrm{min} / 1.73 \mathrm{~m}^{2}$ los cambios no fueron significativos ${ }^{16}$. En nuestro trabajo de pacientes con $\mathrm{FG}<15 \mathrm{ml} / \mathrm{min} / 1.73 \mathrm{~m}^{2}$, se halló una disminución leve pero significativa del FG. Pero en otro estudio previo nuestro, realizado en pacientes con ERC estadio 3B y 4, con DBP y cetoanálogos, se observó, al igual que en el estudio mencionado previamente, un aumento significativo del $\mathrm{FG}^{17}$.

Según Li et al., el uso de cetoanálogos con DBP contribuye a mantener el estado nutricional estimado por albuminemia y colesterol sérico ${ }^{16}$. En nuestro caso, los pacientes recibieron DBP y cetoanálogos y obtuvimos el mismo resultado respecto del estado nutricional estimado por albuminemia e IMC. Los autores refieren que los pacientes que recibieron cetoanálogos mostraron una mejoría significativa en el control del fósforo. Sin embargo, en el análisis de subgrupo, los pacientes que presentaron tasas de $\mathrm{FG}<18 \mathrm{ml} / \mathrm{min} / 1.73 \mathrm{~m}^{2}$ mostraron niveles de fósforo sérico más bajos, hallazgo similar a nuestros resultados en el subgrupo de pacientes mayores de 65 años.

Una reciente investigación realizada en China incorporó pacientes que presentaban una eTFG de menos de $10 \mathrm{ml} / \mathrm{min} / 1.73 \mathrm{~m}^{2}$. Al comenzar una DBP suplementada con cetoanálogos, la eTFG basal media fue de $6.7 \mathrm{ml} / \mathrm{min} / 1.73 \mathrm{~m}^{2}$, que es menor a nuestro caso.
La población estudiada se centró en aquellos pacientes con una función renal inicial extremadamente baja, que han sido menos examinados en otras investigaciones. Después de un año de seguimiento, este estudio encontró que el tratamiento con DBP suplementada con cetoanálogos podría retrasar el inicio de la diálisis ${ }^{18}$.

De manera similar, en un estudio clínico aleatorizado que incluyó a 112 pacientes mayores de 70 años y próximos a la diálisis con eTFG 4-6 ml/min/1.73 m², demostraron que el inicio de la diálisis podría retrasarse de forma segura en un grupo tratado con DMBP $(0.3 \mathrm{~g} / \mathrm{kg} / \mathrm{d})$ suplementada con cetoanálogos, en comparación con los del grupo control (diálisis) ${ }^{19}$. En nuestro estudio el subgrupo de pacientes mayores de 65 años inició tratamiento con DBP $(0.6 \mathrm{~g} / \mathrm{kg} / \mathrm{d})$ suplementada con cetoanálogos y la eTFG inicial entre 11$14.10 \mathrm{ml} / \mathrm{min} / 1.73 \mathrm{~m}^{2}$.

Un estudio de cohorte que utilizó una base de datos longitudinal creada por el Instituto Nacional de Investigación en Salud de Taiwán (NHRI) presenta resultados de 1,483 pacientes con ERC en estadio 5 sin diálisis. La edad media fue de $62.0 \pm 12.9$ años, el $44.5 \%(n=660)$ fueron hombres, el $26.5 \%(n=393)$ presentaron diabetes mellitus (DM), el 59.7\% $(n=885)$ eran hipertensos (HTA) y el $4 \%(n=59)$ presentaron síndrome nefrótico. Todos recibieron una DBP suplementada con cetoanálogos a razón de 1-2 comprimidos dos veces al día (dosis máxima seis comprimidos). Los autores concluyeron que se puede postergar el inicio de la diálisis cuando se utilizan dosis adecuada de Ketosteril ${ }^{\circledR}$ (más de 5.5 comprimidos por día $)^{20}$.

Garneata et al. ${ }^{21}$ mostraron que una terapia dietética con DMBP suplementada con cetoácidos en pacientes con ERC de moderada a avanzada $\left(<30 \mathrm{ml} / \mathrm{min} / 1.73 \mathrm{~m}^{2}\right)$ podría retardar la disminución de la eTFG y mejorar las anomalías metabólicas. Del mismo modo, otros estudios y metaanálisis en pacientes con ERC en estadios (3B,4 y 5) con DMBP o DBP suplementadas con cetoanálogos muestran que podrían retrasar la progresión a la enfermedad crónica terminal ${ }^{17,20}$.

Una de las debilidades de nuestro estudio fue la falta de un grupo control no permitiera evaluar los resultados. Del mismo modo, sería importante la inclusión de un número mayor de pacientes para poder analizar mejor los subgrupos según edad y etiología.

Este trabajo es una de las primeras experiencias reportadas en Hispanoamérica con el uso de cetoanálogos en ERC en estadio 5. 


\section{Conclusión}

En este análisis de datos de pacientes con ERC estadio 5 seguidos por un equipo interdisciplinario de nefrólogos y nutricionistas (con formación en el área nefrológica) en forma periódica, la intervención con DBP suplementada con aminoácidos esenciales y cetoanálogos permitió sostener el tratamiento conservador manteniendo el estado nutricional y equilibrio mineral.

\section{Financiamiento}

Los autores declaran que no hubo financiamiento para la realización del presente trabajo.

\section{Conflicto de intereses}

Aimar M. Andrea tiene contrato de trabajo con Nutrihome S.A. El resto de los autores declara no tener conflicto de intereses en relación con el artículo publicado.

\section{Responsabilidades éticas}

Protección de personas y animales. Los autores declaran que para esta investigación no se han realizado experimentos en seres humanos ni en animales.

Confidencialidad de los datos. Los autores declaran que han seguido los protocolos de su centro de trabajo sobre la publicación de datos de pacientes.

Derecho a la privacidad y consentimiento informado. Los autores declaran que en este artículo no aparecen datos de pacientes.

\#Colaboradores del Grupo RIANA (Red Interdisciplinaria de Atención Nutricional Ambulatoria): Dora Aguero, Fresenius Medical Care Moreno-Mercedes Alba, Centro de Nefrología Cipolletti, Cipolletti-Río Negro-M. Elisa Armendariz, Instituto del Riñón Neuquén-Jorge L.Audisio, Fresenius Medical Care y Hospital Interzonal Dr. José Penna, Bahía Blanca-Ma. Elena Biaiñ, I.U. Cemic-Sergio Boni, Aterym Alta Gracia-Florencia Contard, Hospital Comunitario Generalista Evita, Santa Rosa, La Pampa-Silvia Cristóbal, CENDIAL-NEXDIAL Centro de Nefrología y Diálisis-Priscila de Feo, Nutrihome S.A., Ciudad Autónoma de Buenos Aires-Damián Díez, Nefrología Coorporación Médica de San Martín, Pcia. de BS AS-Adriana Fernández, Hospital de Niños de La Plata-Gustavo Ferricher, Sanatorio Mater Dei-Santiago Fonseca, Servicio de Diálisis Riccobelli, Santa Fe-David Gluz, Servicio de Diálisis Riccobelli, Santa
Fe-Horacio González, Hospital de Niños de La Plata/ IDIP CIC-PBA-Pedro Guerrero, Instituto del Riñón Neuquén-Horacio Iros, CENDIAL-Ramiro Korsunsky, Fresenius Medical Care y Hospital Interzonal Dr. José Penna, Bahía Blanca-Gustavo Laham, I.U. Cemic-Andrés Martinuzzi, Nutrihome S.A, Neuquén-Pablo Mele, Hospital Interzonal Dr. José Penna y Centro de Diálisis del Sur, Bahía Blanca-Ma. Cristina Milano, Freseinus Medical Care-Marcela Munizaga, Aterym Córdoba-Romina B. Philippi, Fresenius Medical Care Hospital Italiano-Malvina Ravazio, Fresenius Medical Care Moreno-Mariana Sanguineti, Nutrihome S.A., Ciudad Autónoma de Buenos Aires-César San Martín, Nefrología Coorporación Médica de San Martín, Pcia. de BS AS-Noelia Schoeffer, ITAC By Diaverum, Consultorios Externos-Diego Serra, Cedex y Hospital Lucio Molas Santa Rosa, La Pampa-Sleiman Jihan, I.U. Cemic-Mario Traverso, Centro de Nefrología Cipolletti, Cipolletti, Río Negro-Adriana Urso, Nutrihome S.A., Ciudad Autónoma de Buenos Aires-Gabriela Zulueta, Cedex, Santa Rosa, La Pampa-Ma. Victoria Fasano, Departamento de Matemática, IDIP La Plata.

\section{Bibliografía}

1. Kalantar-Zadeh K, Joshi S, Schlueter R, Cooke J, Brown-Tortorici A, Donnelly $\mathrm{M}$, et al. Plant-dominant low-protein diet for conservative management of chronic kidney disease. Nutrients. 2020;12(7):1931.

2. Segunda Encuesta Nacional de Nutrición y Salud, ENNyS 2, 2019 [Internet]. Ministerio de Salud Nación Argentina-2019. Disponible en: https:// slanh.net/resultados-preliminares-de-la-segunda-encuesta-nacional-desalud-y-nutricion-e-n-n-y-s-2-2018-2019-prevalencia-de-enfermedad-renal-cronica-en-argentina

3. Marinovich S, Bisigniano L, Hansen Krogh D, Celia E, Tagliafichi V, Rosa Diez G, et al.: Registro Argentino de Diálisis Crónica SAN-INCUCAI 2018 [Internet]. Buenos Aires, Argentina: Sociedad Argentina de Nefrología e Instituto Nacional Central Único Coordinador de Ablación e Implante-2019. Disponible en: https://www.renal.org.ar/13-registro_dialisis/2017-18/01-2018.pdf

4. Fouque D, Ikizler TA. Implementing low protein diets in clinical practice in patients with chronic kidney disease. Nephrol Dial Transplant. 2020;35(10):1643-5.

5. Ikizler TA, Burrowes JD, Byham-Gray LD, Campbell KL, Carrero JJ, Chan W, et al. KDOQI Clinical Practice Guideline for Nutrition in CKD: 2020 Update. Am J Kidney Dis. 2020;76(3 Suppl 1):S1-S107.

6. Bellizzi V, Bianchi S, Bolasco P, Brunori G, Cupisti A, Gambaro G, et al. A Delphi consensus panel on nutritional therapy in chronic kidney disease. J Nephrol. 2016;29:593-602.

7. Cuppari L, Nerbass FB, Avesani CM, Kamimura MA. A practical approach to dietary interventions for nondialysis-dependent CKD patients: the experience of a reference nephrology center in Brazil. BMC Nephrol. 2016;17(1):85.

8. Aparicio M, Bellizzi B, Chauveau P, Cupiste A, Ecder T, Fouque D, et al. Protein-restricted diets plus keto/amino acids - A valid therapeutic approach for chronic kidney disease patients. J Ren Nutr. 2012;22:S1-21.

9. Mitch WE, Remuzzi G. Diets for patients with chronic kidney disease, should we reconsider? BMC Nephrol. 2016;17(1):80.

10. Di Micco L, Di Lullo L, Bellasi A, Di lorio BR. Very low protein diet for patients with chronic kidney disease: Recent insights. J Clin Med. 2019;8(5):718.

11. Jiang Z, Zhang X, Yang L, Li Z, Qin W. Effect of restricted protein diet supplemented with keto analogues in chronic kidney disease: a systematic review and meta-analysis. Int Urol Nephrol. 2016;48:409-18.

12. Garneata L, Mircescu G. Effect of low-protein diet supplemented with keto acids on progression of chronic kidney disease. J Ren Nutr. 2013;23:210-3. 
13. Shah AP, Kalantar-Zadeh K, Kopple JD. Is there a role for ketoacid supplements in the management of CKD? Am J Kidney Dis. 2015;65:659-73.

14. Levey S, Bosch J, Breyer L, Greene T, Rogers N, Roth D. A more accurate method to estimate glomerular filtration rate from serum creatinine: a new prediction equation. Ann Intern Med. 1999;130:461-70.

15. Organización Mundial de la Salud. Obesidad y Sobrepeso. Datos y cifras. 2018 [Internet]. Organización Mundial de la Salud-2018. Disponible en: https://www.who.int/es/news-room/fact-sheets/detail/obesity-and-overweight

16. Li A, Lee HY, Lin YC. The effect of ketoanalogues on chronic kidney disease deterioration: A meta-analysis. Nutrients. 2019:11(5):957.

17. Aimar MA, Pomiglio G, Baccaro F, Traverso M, Audisio J, De Feo P et al. Progression of renal function in patients with chronic kidney disease on a low-protein diet supplemented with aminoacids and ketoanalogues. Nutr Hosp. 2018;35(3):655-60.
18. Yen CL, Fan PC, Lee CC, Kuo G, Tu KH, Chen JJ, et al. Advanced chronic kidney disease with low and very low GFR: Can a low-protein diet supplemented with ketoanalogues delay dialysis? Nutrients. 2020;12(11):3358

19. Brunori G, Viola BF, Parrinello G, De Biase V, Como G, Franco V, et al. Efficacy and safety of a very-low-protein diet when postponing dialysis in the elderly: a prospective randomized multicenter controlled study. Am J Kidney Dis. 2007:49(5):569-80.

20. Wu CH, Yang YW, Hung SC, Kuo KL, Wu KD, WuVC, et al.-National Taiwan University Study Group on Acute Renal Failure (NSARF). Ketoanalogues supplementation decreases dialysis and mortality risk in patients with anemic advanced chronic kidney disease. 2017;12(5):e0176847.

21. Garneata L, Stancu A, Dragomir D, Stefan G, Mircescu G. Ketoanalogue-supplemented vegetarian very low-protein diet and CKD progression. J Am Soc Nephrol. 2016;27(7):2164-76. 